\title{
Split thickness skin grafts (STSGs) reflect differences in early composition of extracellular matrix on wound bed year later in proteomic analysis - a comparative study
}

\author{
Heli Lagus, Mariliis Klaas, Susanna Juteau, Outi Elomaa, \\ Juha Kere, Jyrki Vuola, Viljar Jaks and Esko Kankuri \\ Department of Plastic Surgery, Helsinki University Hospital, Töölö Hospital, POB 266, FIN-00029, Finland
}

\section{Study design}

The aim of this study was to investigate if the different wound bed compositions would result alterations in protein expression in the transplanted split thickness skin grafts (STSGs) one year later.

Large deep burns (22-45\% TBSA) of four adult patients from Helsinki Burn Centre were excised after injury. Three $5 \mathrm{~cm} \times 10 \mathrm{~cm}$ consecutive areas were covered with: STSG (Control), an artificial dermal template (Integra $\AA$ ) and a temporary cover viscose cellulose sponge (Cellonex ${ }^{\mathrm{TM}}$ ) inducing granulation tissue formation.

The findings were reflected to samples of patients with psoriasis and healty control.

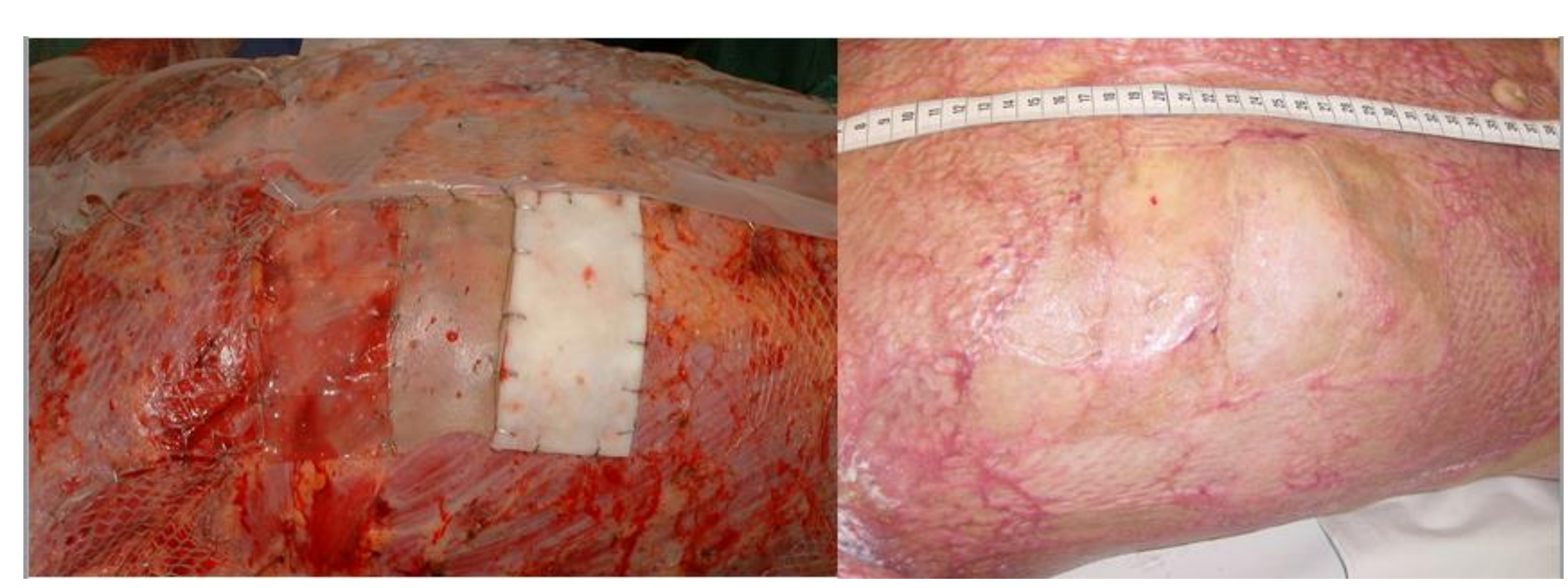

Treatment areas from left to right: Integra- STSG-Cellonex at the operation and 3 months later.

\section{Results and Discussion}

Proteomics analysis resulted in identification of 481 proteins. $12 \%$ of these proteins were significantly differently expressed across treatments: 30 proteins in the epidermis and 24 proteins in the dermis.

Statistically significant differently expressed proteins: In epidermis:

DNAH10: both treatments, Integra $\AA$ and Cellonex ${ }^{\mathrm{TM}}$ increasing wound bed ECM content before transplanting STSGs showed immensely higher expression of DNAH10 in STSGs compared to control sites,

the protein profile in epidermis of STSGs of both treatment groups (dermal template and cellulose sponge) seem to be more similar with each other than with the control group

Of the most differently expressed 7 proteins of epidermis STSGs on Integra $\AA$ showed the least expression in 5/7 and STSGs of control group 6/7 the most expression

\section{In dermis:}

four cytokeratins (KRT1, KRT6C, KRT16 and KRT77) expressed differently in dermis, most likely deriving from the adnexes

all of them most expressed in STSGs on cellulose sponge induced granulation tissue

3 out of 4 expressed the least in STSGs on dermal template

Cytokeratins 6 and 16 known to be upregulated during keratinocyte activation and in hyperproliferative epidermis

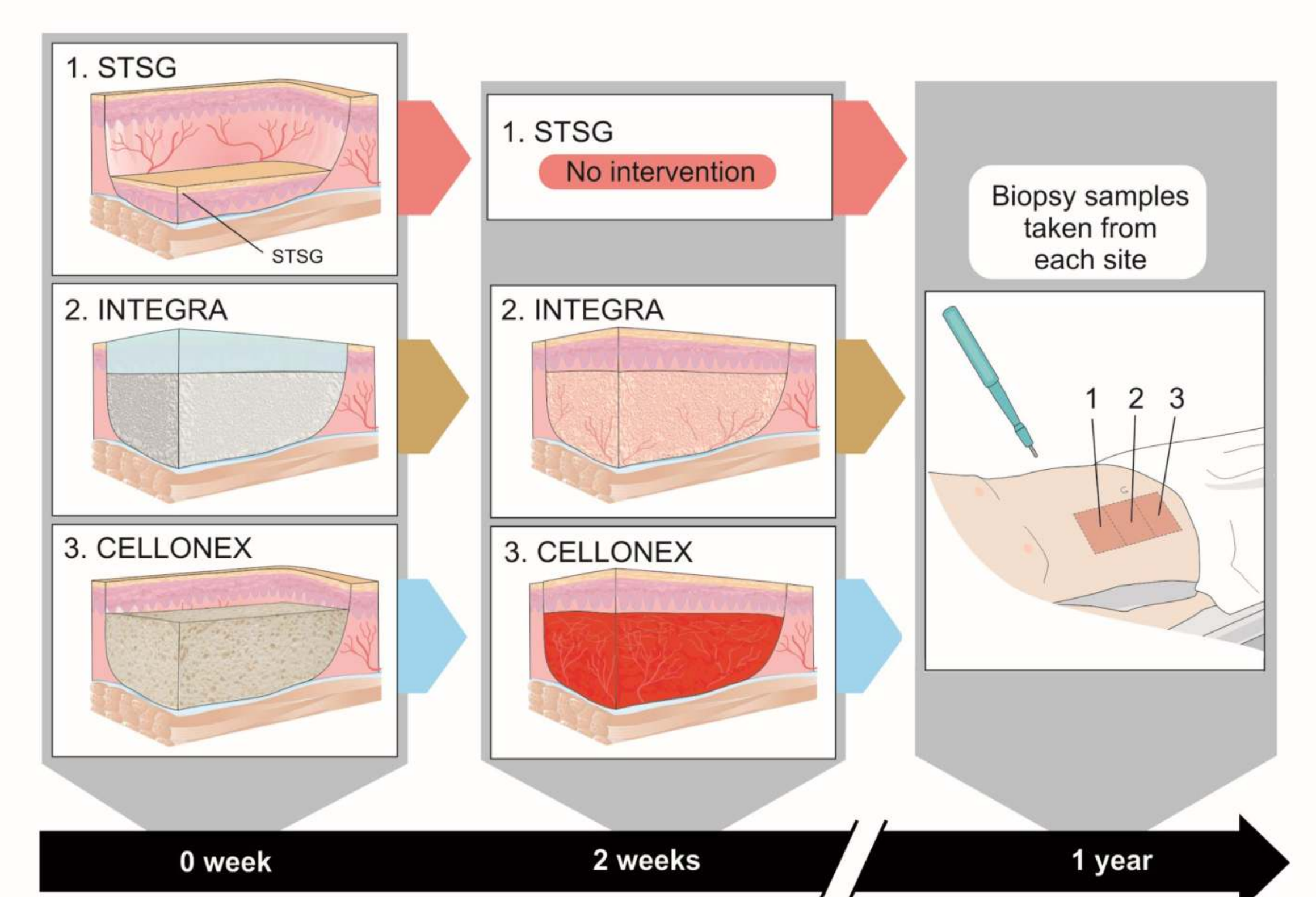

STSG as the Control group was grafted immediately after excision. Two weeks after primary operation the wound beds treated with Integra ${ }^{\circledR}$ and Cellonex ${ }^{\mathrm{TM}}$ were covered with STSGs.

DNAH1O - the most differently expressed protein

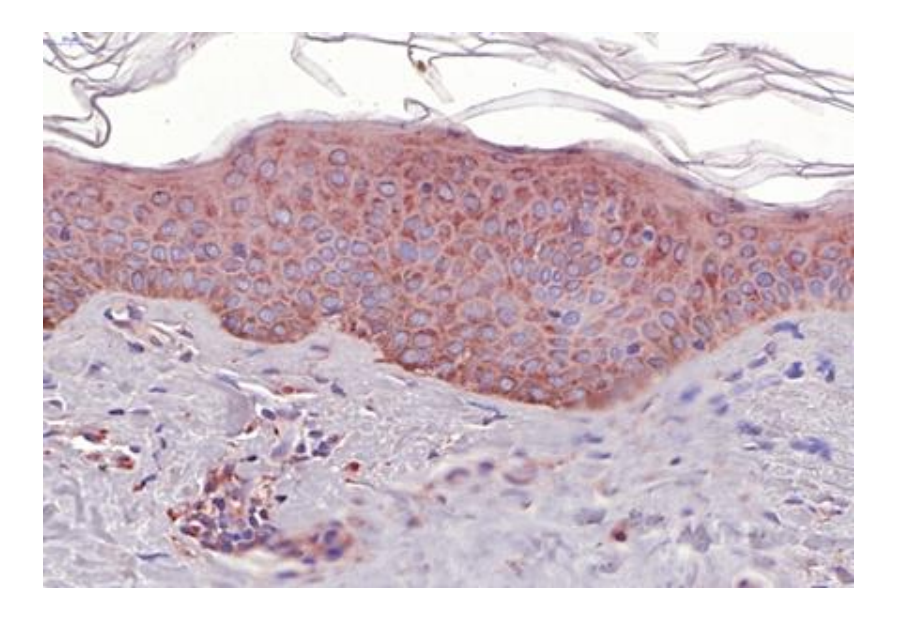

Integra $8+$ STSG

DNAH1O - in healthy control and in psoriasis

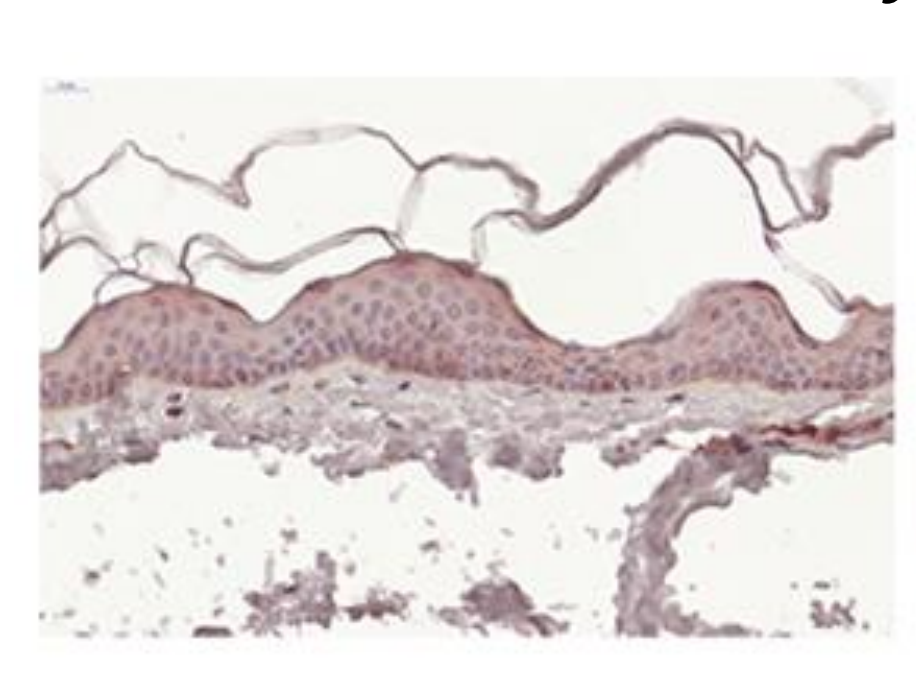

control

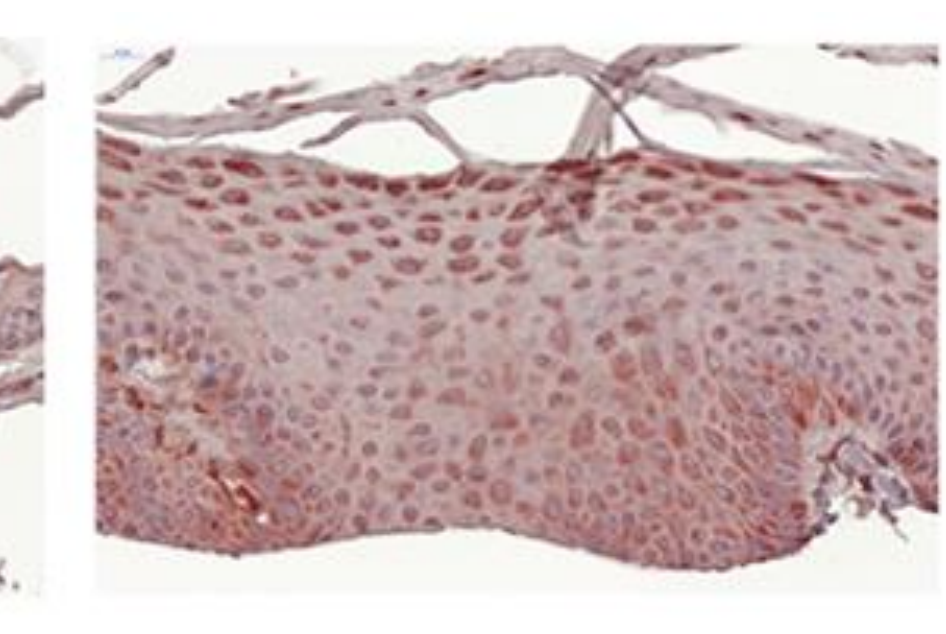

ps lesion

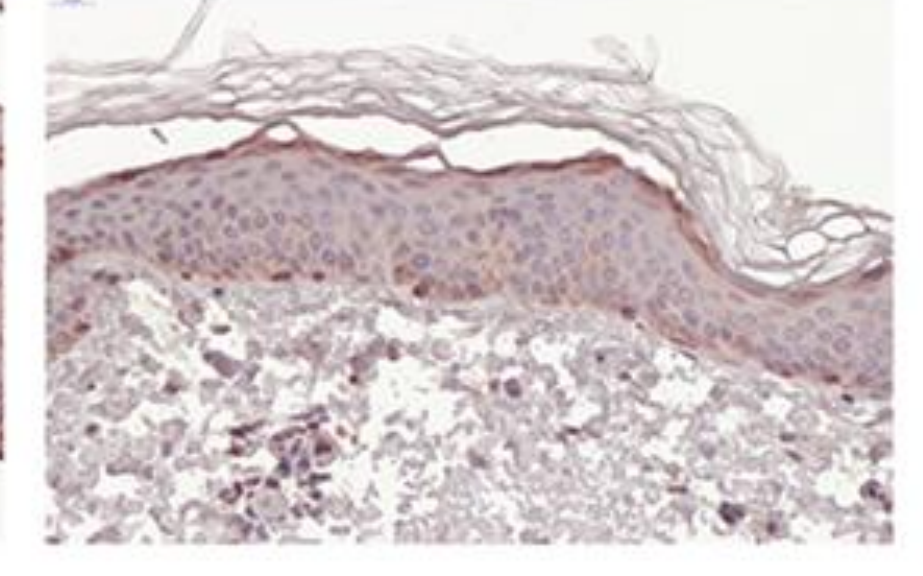

ps healthy
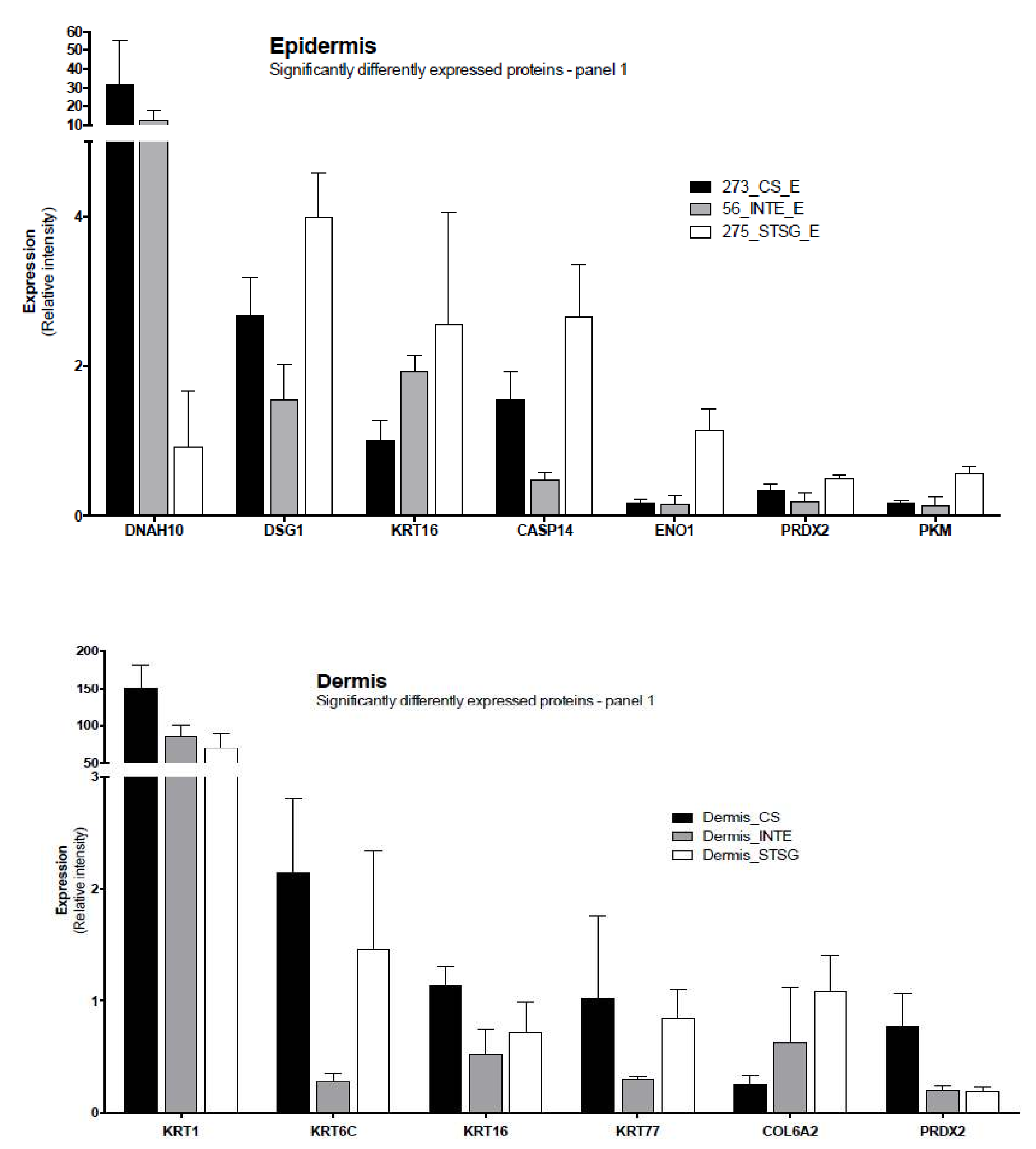

Our findings suggest that Integra ${ }^{\circledR}$ provides less active or less supportive microenvironment for keratinocyte proliferation than the other groups Type XII collagen interacts with type I collagencontaining fibrils; Integra contains type I collagen which might explain the higher expression of collagen type XII in STSGs on Integra compared to STSGs on other groups

Clinically and histologically the differences between the different treatment groups were minimal one year after excision

\section{Samples and analyses}

$3 \mathrm{~mm}$ punch biopsies were collected at each site at one-year follow-up. Epidermis and dermis were collected separately using laser-capture microdissection from paraffin-embedded sections of tissue samples. Samples were analyzed by massspectrometry and data was quantified with MaxQuant 1.4.0.8 software package. Statistical differences in protein expression both in epidermis and dermis between the treatment areas were assessed by a twotailed t-test.

ImageJ software was used for image analysis. The findings were validated with immunohistochemistry of selected proteins (DNAH10 and CASP14).

DNAH10 epidermis/ 12 months

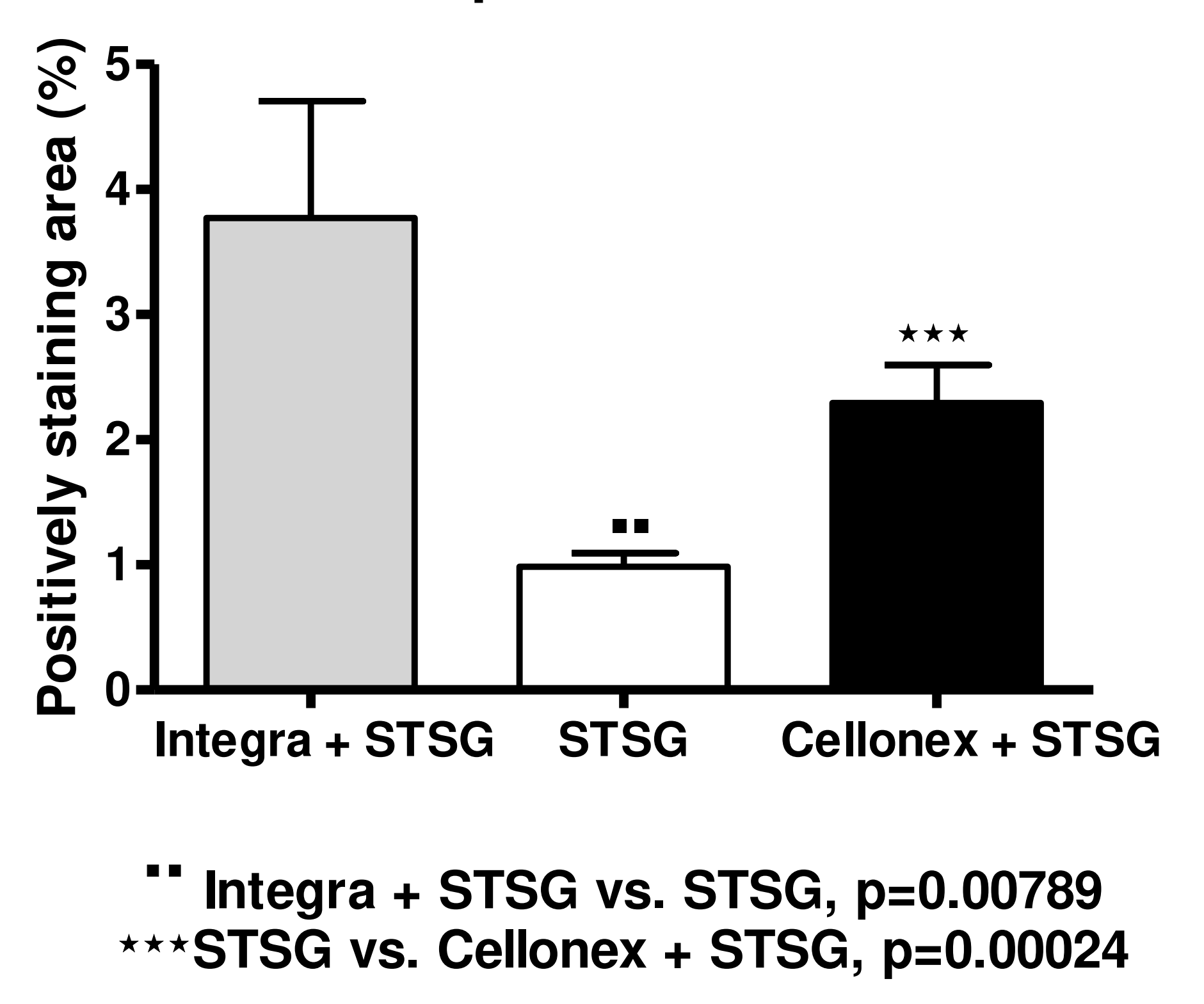

Heavy chain axonemal dynein 10 (DNAH10) belongs to family of cytoskeletal microtubule-associated motor proteins, dyneins, that generate sliding of microtubules using ATP as a source of energy. Dyneins in general have roles e.g. in pigment transport in melanophores and in transporting nutrients. The role of DNAH10 in skin is not known.

\section{Conclusions \\ - Different wound bed compositions have long- lasting or even permanent effects on STSGs \\ - 1 year after the operation 54 proteins were significantly differently expressed in STSGs \\ - DNAH10 expression was found to respond to wound bed preparation in STSGs \\ - DNAH10 expression is increased in psoriasis lesions \\ - Further studies needed to elucidate significance and function of DNAH10}

\section{Acknowledgements}

We thank Lahja Eurajoki and Maria Karhu for assistance and Maarit Sarlomo-Rikala and Tom Böhling for earlier analysis of the samples.

The study has been supported by the Scientific Committee of the Finnish Ministry of Defence.

\section{References}

H Lagus, M Sarlomo-Rikala, T Böhling, J Vuola, Prospective study on burns treated with Integra, a cellulose sponge and split thickness skin graft - Comparative clinical and histological study, Burns 39 (2013)

H Lagus, E Kankuri, K Nuutila, S Juteau, M Sarlomo-Rikala, J Vuola, Induced granulation tissue but not artificial dermis enhances early host-graft interactions in full thickness burn wounds, World Journal of Surgery accepted to be published 